\title{
Single Beacon Navigation: Localization and Control of the MARES AUV
}

\author{
Bruno Ferreira, Aníbal Matos, Nuno Cruz \\ INESC Porto and University of Porto \\ Rua Dr. Roberto Frias, 378 \\ 4200 - 465 Porto, Portugal \\ $\{$ bm.ferreira, anibal, nacruz $\} @$ fe.up.pt
}

\begin{abstract}
This paper addresses the simultaneous localization and control of an AUV using a single acoustic beacon. To determine its horizontal position, the AUV fuses distances to the single beacon with dead reckoning data, heading and longitudinal velocity. A particle filter and an extended Kalman filter are implemented and compared in terms of performances. As this is an ill-posed problem, special guidance laws are derived so that the overall horizontal positioning error remains bounded. Besides presenting the derivation of such guidance and control laws, as well as procedures to estimate the horizontal position, we also demonstrate the performance of the proposed system by means of simulation results.
\end{abstract}

\section{INTRODUCTION}

Autonomous vehicles and robots have been used in many applications over the last years addressing the need for automatic systems in order to improve performance or safety, for example. In this sense, robotics is a challenging domain in which several engineering subjects have to be considered to build robust and efficient systems. Adequate motion of these vehicles (or robots) requires the knowledge about the current position which, in turn, implies the use of sensors to capture environment data. Nowadays, devices like GPS, Doppler based sensors or Inertial Measurement Unit (IMU) provide accurate measurements of the motion.

However, GPS are not available for underwater environments and Doppler effect sensors and INS are affected by biases that are difficult to estimate, contributing to positioning errors that grow with time or traveled distance. In this sense, underwater vehicle navigation systems are often aided by measurements of ranges [1]-[5] to beacons or markers. Nevertheless, this implies the need for knowledge of marker position or deployment of several setup material, which is intended to be minimal.

This paper focuses on navigation of underwater vehicles based on measurements of ranges to a single beacon. We address the problem of navigation and control in a coupled way in order to guarantee observability.

Some works have been performed in single beacon navigation [1], [3]. Both implementations include Kalman filter estimation, being different on the initialization process. Their simulation and practical results show successful operation of the navigation system.

Bruno Ferreira was suported by the Portuguese Fundation for Science and Technology through the PhD grant SFRH/BD/60522/2009.
The subject addressed here may be directly adapted to several scenarios. In particular, for underwater vehicles, one may imagine several scenarios like the search of a lost object equipped with an emitter or the so-called homing of an underwater vehicle in a moving platform. This last scenario is of utmost importance, considering that it may be applied to recover a vehicle: if for any reason, a vehicle fails during its mission or loses itself due to issues in sensors or in the navigation system, it may switch to a safety mode in witch it tries to find an emitter in a surface vehicle or in a well known place, for instance.

Recently, the inclusion of acoustic modems in underwater systems is becoming common. Generally, underwater systems embody two modems: one in the vehicle and another on a base station. Further to their role as a mechanism to exchange data, these devices also provide the distance between them, in real time. By taking advantage of these precise range measurements, it is then possible to navigate accurately without requiring any other beacons.

\section{THE PROBLEM}

Throughout this paper, we will consider the Autonomous Underwater Vehicle (AUV) MARES. This vehicle was developed at FEUP (Faculty of Engineering of the University of Porto) where its performances were already demonstrated in previous works [6]-[8]. Beyond other characteristics, the MARES AUV has the particular abilities to immobilize in

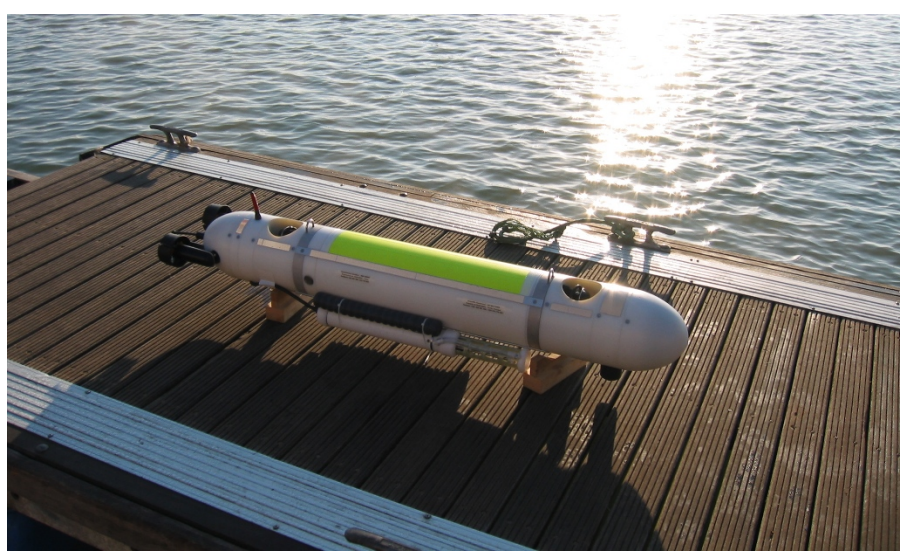

Fig. 1: Autonomous underwater vehicle MARES. 
the column water and to dive independently of the forward velocity. It is endowed by four thrusters: the vertical motion (translation and rotation) relies on two propellers inserted in the hull while the horizontal motion is ensured by two propellers placed on the rear part of the vehicle.

Typical missions of MARES include environment sampling and monitoring in which the motion of the vehicle is needed. A set of equipment such as fluorometers, turbidity, CTD (conductivity, temperature, depth) sensors are conveyed on board. Angular variables are measured by a compass and tilt sensors, while depth is indirectly measured by a pressure sensor. These outputs are fairly precise, accurate and noiseless. Nevertheless, horizontal localization does not profit of the same advantage. The current solution for navigation of MARES contemplates two acoustic beacons deployed on the surface of the region of interest. Using a long baseline (LBL) [7], the horizontal position of the vehicle is estimated through combination of distances to each of the beacons and dead-reckoning computation. Distances are derived by the calculation of the time-of-flight of acoustic pulses emitted by the beacons. Errors of the position estimation are typically in the range of 1 to 2 meters.

In order to minimize the set-up deployment, specially acoustic beacons, we aim at developing a solution that allows for robust navigation with a single beacon. In this work, we consider the particular case of homing. This task is useful for applications in which the AUV has to be recovered from the water, for example. Even in scenarios where the localization of the vehicle is not an issue, the homing approach may be interesting to the vehicle meet the beacon.

To analyze the motion of the MARES AUV, we will use the same notation as in [9]. The position and angles of the vehicle are expressed through the vector $\eta_{c}=[x, y, z, \phi, \theta, \psi]^{T} \in \mathbb{R}^{6}$ in the inertial frame $\{\mathcal{I}\}$. The pair $(x, y)$ define the horizontal position of the vehicle, $z$ the depth at which the vehicle is and $\phi, \theta, \psi$ are the roll, pitch and yaw angles, respectively. The velocities of translation and rotation, related to the fluid, are given by the vector $\nu_{c}=[u, v, w, p, q, r]^{T} \in \mathbb{R}^{6}$, expressed in the body frame $\{\mathcal{B}\}$. It is assumed that the fluid is irrotational and that only the horizontal components of velocity (water current) are non-null. Hence, the fluid velocity vector is given, in the inertial frame $\{\mathcal{I}\}$ by $\nu_{f c}=\left[v_{x}, v_{y}, \mathbf{0}_{1 \times 4}\right]^{T} \in \mathbb{R}^{6}$. Suppose there is linear transformation matrix $J_{c}(\eta) \in \mathbb{R}^{6 \times 6}$ dependent on the angular position of the vehicle that maps vectors in $\{\mathcal{B}\}$ to $\{\mathcal{I}\}$. The motion dynamics is described by the following system:

$$
\begin{aligned}
\dot{\eta}_{c} & =J_{c}\left(\eta_{c}\right) \nu_{c}+\nu_{f c} \\
M \dot{\nu}_{c} & =-\left(C\left(\nu_{c}\right)+D\left(\nu_{c}\right)\right) \nu_{c}+g\left(\eta_{c}\right)+P f_{p}
\end{aligned}
$$

where $M \in \mathbb{R}^{6 \times 6}$ is the inertia matrix, $C\left(\nu_{c}\right) \in \mathbb{R}^{6 \times 6}$ is the Coriolis and centripetal terms matrix, $D\left(\nu_{c}\right) \in \mathbb{R}^{6 \times 6}$ is the viscous damping matrix, $g\left(\eta_{c}\right) \in \mathbb{R}^{6}$ is the restoring forces and moments vector, $P \in \mathbb{R}^{6 \times n}$ is the actuator distribution matrix, where $n$ is the number of motion actuator, and $f_{p} \in \mathbb{R}^{n}$ is the vector of actuator forces whose elements are the forces applied by each actuator. Further information and derivation of these terms may be found in [9], [10] or, for the particular case of MARES, in [6].

For simplicity, instead of considering the complete model, several authors consider the motion of underwater vehicles in a decoupled way [9], [10]. In our previous work [6], we developed controllers that give to MARES the ability to move independently in vertical and horizontal planes. This feature allows us to be concerned only with the horizontal motion for this paper. A reduced model of the dynamics (1), (2) is used instead of the complete model (see [6]), in which $\eta=$ $[x, y, \psi]^{T}, \nu=[u, v, r]^{T}$ and $\nu_{f}=\left[v_{x}, v_{y}, 0\right]^{T}$ are the reduced vectors of position and velocities in the horizontal subspace. The motion dynamics takes the same form as in (1) and (2).

Assuming that the inner loop (velocity loop) of the system is closed and stable, we are able to give dynamic references to velocity controllers. This approach was already implemented and its operation was successfully demonstrated [6]. It allows us to simplify our analysis and to abstract from the complex motion dynamics. Hence, we assume that we can manipulate the state variable $u$ and $r$ by setting the references $u_{r e f}$ and $r_{r e f}$ on the velocity controller. Let's consider the origin of the inertial frame $\{\mathcal{I}\}$ to be coincident with the beacon on the surface. Its $x$-axis coincides with the north direction while the $y$-axis coincides with the east direction. The kinematics of the reduced model in (1) can be simply written as

$$
\begin{aligned}
\dot{\eta}=\left[\begin{array}{c}
\dot{x} \\
\dot{y} \\
\dot{\psi}
\end{array}\right] & =\left[\begin{array}{ccc}
\cos (\psi) & -\sin (\psi) & 0 \\
\sin (\psi) & \cos (\psi) & 0 \\
0 & 0 & 1
\end{array}\right]\left[\begin{array}{l}
u \\
v \\
r
\end{array}\right]+\left[\begin{array}{c}
v_{x} \\
v_{y} \\
0
\end{array}\right] \\
& =J(\eta) \nu+\nu_{f},
\end{aligned}
$$

assuming that roll and pitch angles are null, $\phi=\theta=0$.

Hence, homing is equivalent to stabilize the origin of $(x, y)$ in (3) through control laws $u=\gamma_{u}(\eta)$ and $r=\gamma_{r}(\eta)$.

However, the pair $(x(t), y(t))$ is unknown and has to be estimated in order to obtain an apropriate behavior while approaching the origin. The available measurements are the range to the beacon $R$, the yaw angle $\psi$ and the depth $z$. While the two later variables are reasonably noiseless, the range is not:

$$
R=\sqrt{x^{2}+y^{2}+z^{2}}+w_{n},
$$

where $w_{n}$ is a non-null mean, non-gaussian noise variable, dependent on the amplitude of $R$, affected by multipath of acoustic waves and interferences [4].

Part of the homing problem can be solved by estimating $\hat{x}, \hat{y}, \hat{v}_{x}$ and $\hat{v}_{y}$. To achieve so, particle filter and Kalman filter are methods that have been applied in several robotic localization problems. However, in our case, due to questions of observability, it is expected that the control laws and the estimation cannot be decoupled. The control law has to take into account the confidence on the estimates while it generates trajectories that ensure stability. The next section describes our implementation, discussing some of the major questions of estimation and control. 


\section{NAVIGATION}

A good navigation system is crucial to provide vehicle localization and positioning, with strong impact on mission performance. By measuring the time of flight of the acoustic waves, it is possible to determine the distance between the vehicle and the beacon. Nevertheless, this measurement only provides information along one dimension. Thus it is aided by a compass and a pressure sensor that makes it possible to observe orientation and depth.

Localization is reduced to an estimation problem in which Kalman filters and particle filters seem to be the most promising approaches. In order to verify and compare the two methods, we developed both. Their formulations are shown in the following subsections while initialization and control questions are tackled in the last two.

\section{A. Estimating the position: extended Kalman filter}

Kalman filter is an optimal estimator for linear systems affected by white noise in measurements and state. Its operation for nonlinear system with gaussian noise was classified as suboptimal [11]. However, it has been implemented in several works ( see [1], [2], [4], for example) where the results are convincent in terms of performances, even in the presence of non-gaussian, non-null mean noise. This first approach contemplates an extended Kalman filter (EKF) to estimate the horizontal position $(\hat{x}, \hat{y})$ and the water current velocities $\left(\hat{v}_{x}, \hat{v}_{y}\right)$.

We assume that the state variable $\psi$ is directly obtained from the compass and $z$ is obtained from the pressure sensor. Due to low noise of these sensor outputs, we consider that filtering is not needed, which makes possible their direct inclusion in (3) and (4). The use of a computational system implies discretization of time. The step interval in a typical mission with MARES is $\Delta t=100$ milliseconds. At each time step, the values of depth and compass are updated. However, because of the natural propagation of acoustic waves in the water, the update of the range is performed at lower frequency: generally the overall acoustic system is set to a frequency of $0.5 \mathrm{~Hz}$. Note that the reception of ping may fail due to occlusions, interferences or reflections in non-homogeneous environments. For the extended Kalman filter formulation (see [11]), let the measurement at instant $t_{k}$ be given by

$$
m_{k}=R\left(t_{k}\right)=h_{k}\left(\eta_{k}\right)+v_{k},
$$

where $h_{k}\left(\eta\left(t_{k}\right)\right)=\sqrt{x\left(t_{k}\right)^{2}+y\left(t_{k}\right)^{2}+z\left(t_{k}\right)^{2}}$ and $v_{k} \sim$ $\mathcal{N}\left(0, R_{m}\right)$ is assumed to be a noise variable that follows a normal distribution with variance $R_{m}$. As it was already stated, this assumption is not true but, for this formulation, we will assume so.

For precise localization, it is important to estimate the water current for inclusion in dead-reckoning computation. In Kalman filter formulation, it implies to include the current components as state variables. Assuming they are constant, from (3), we can easily derive:

$$
\overbrace{\left[\begin{array}{c}
\dot{x} \\
\dot{y} \\
\dot{v}_{x} \\
\dot{v}_{y}
\end{array}\right]}^{\dot{X}}=\overbrace{\left[\begin{array}{cccc}
0 & 0 & 1 & 0 \\
0 & 0 & 0 & 1 \\
0 & 0 & 0 & 0 \\
0 & 0 & 0 & 0
\end{array}\right]\left[\begin{array}{c}
x \\
y \\
v_{x} \\
v_{y}
\end{array}\right]+\left[\begin{array}{cc}
\cos (\psi) & -\sin (\psi) \\
\sin (\psi) & \cos (\psi) \\
0 & 0 \\
0 & 0
\end{array}\right]\left[\begin{array}{c}
u \\
v
\end{array}\right](6)}^{f(X)}
$$

where $w \in \mathbb{R}^{4} \sim \mathcal{N}(0, Q)$ is the noise vector with null mean and variance $Q \in \mathbb{R}^{4 \times 4}$.

The formulation of the extended Kalman filter follows from [11]. Whenever a new measurement $R\left(t_{k}\right)$ is available, the state estimate update is given by

$$
\hat{X}_{k \mid k}=\hat{X}_{k \mid k-1}+K_{k}\left[R_{k}-h_{k}\left(\hat{X}_{k \mid k-1}, z\right)\right]
$$

where $K_{k}$ is the so-called Kalman gain at instant $t_{k}$. This vector is computed through the following expression:

$$
K_{k}=P_{k \mid k-1} H_{k}^{T}\left(H_{k} P_{k \mid k-1} H_{k}^{T}+R_{k}\right)^{-1},
$$

where $H_{k}$ is the Jacobian of $h_{k}$ at point $\hat{X}$ given by

$$
H_{k}=\left[\begin{array}{c}
\partial h_{k} / \partial x \\
\partial h_{k} / \partial y \\
\partial h_{k} / \partial v_{x} \\
\partial h_{k} / \partial v_{y}
\end{array}\right]_{\left.\right|_{X=\hat{X}_{k \mid k-1}}}=\left[\begin{array}{c}
x / h_{k} \\
y / h_{k} \\
0 \\
0
\end{array}\right]_{\mid X=\hat{X}_{k \mid k-1}}
$$

and $P$ is the estimate covariance, which is updated at each new range measurement through

$$
P_{k \mid k}=\left(I-K_{k} H_{k}\right) P_{k \mid k-1} .
$$

These updates occur only when a new measurement is available. However, the range is not observed at each time step and the position estimate has to propagated through deadreckoning. From (1) and (2), one can easily derive the discretized formulation:

$$
\begin{aligned}
{\left[\begin{array}{c}
\hat{x}_{k \mid k-1} \\
\hat{y}_{k \mid k-1}
\end{array}\right]=} & {\left[\begin{array}{l}
\hat{x}_{k-1 \mid k-1} \\
\hat{y}_{k-1 \mid k-1}
\end{array}\right]+} \\
& \left(\left[\begin{array}{cc}
\cos \left(\psi_{k}\right) & -\sin \left(\psi_{k}\right) \\
\sin \left(\psi_{k}\right) & \cos \left(\psi_{k}\right)
\end{array}\right]\left[\begin{array}{l}
\hat{u}_{k-1} \\
\hat{v}_{k-1}
\end{array}\right]+\left[\begin{array}{c}
\hat{v}_{x k} \\
\hat{v}_{y k}
\end{array}\right]\right) \Delta t
\end{aligned}
$$

where $\Delta t=t_{k}-t_{k-1}$. Here, the velocities $\hat{u}$ and $\hat{v}$ are estimated. Based on the model of equation (2), the velocity is dead-reckoned according to the following expression:

$$
\begin{aligned}
\hat{\nu}_{k+1} & =\left[\begin{array}{l}
\hat{u}_{k} \\
\hat{v}_{k} \\
r_{k}
\end{array}\right] \\
& =\hat{\nu}_{k}+M^{-1}\left(-(C+D) \hat{\nu}_{k}+g+P f_{p k}\right) \Delta t
\end{aligned}
$$

where $C=C\left(\hat{\nu}_{k}\right), D=D\left(\hat{\nu}_{k}\right), g=g\left(\phi_{k}, \theta_{k}\right)$. We recall that in (8) and (9), the variable $\psi_{k}$ is directly measured by the compass, from which $r=\frac{d \psi_{k}}{d t}$ is easily derived. Note that in (9), the thruster forces $f_{p}$ are estimated externally. We have to highlight that dead-reckoning relies on the accuracy of the model for velocity estimation. Inaccurate models can strongly degrade the estimation of the state. 
At each dead-reckoning step, the covariance matrix evolves as follow:

$$
P_{k \mid k-1}=F_{k-1} P_{k-1 \mid k-1} F_{k-1}^{T}+Q
$$

where $F$ is the Jacobian of the function $f(X)$ in (6) at point $\hat{X}$ :

$$
F=\left.\frac{\partial f(X)}{\partial X}\right|_{X=\hat{X}}=\left[\begin{array}{llll}
0 & 0 & 1 & 0 \\
0 & 0 & 0 & 1 \\
0 & 0 & 0 & 0 \\
0 & 0 & 0 & 0
\end{array}\right]
$$

One of the major issues with the extended Kalman filter is the initialization. To avoid divergence and guarantee fast convergence of the estimate, the initial positions and water current velocities have to be adequately initialized. This question will be tackled later in subsection III-C. The next subsection present a valid alternative to EKF though it can also be used as an initialization method.

\section{B. Estimating the position: particle filter}

While the extended Kalman filter approach assumes the presence of Gaussian noises on observations and on state propagation, particle filters do not make any assumption on the probability distribution of the noise [12], [13]. In fact, the posterior probability distribution is approximated by a finite number of particles which are vectors whose components represent probable state estimates.

Although particle filter is a promising approach, it is computationally costly when compared with EKF. Generally, the number of particles is large to guarantee good coverage of the belief distribution and grows substancially with the number of state variables to estimate [13]. Once our computational ressources are limited, there is a trade-off between accuracy and processing requeriments. The computational system of MARES manage several critical sensors and actuators whose processing time is considerable. This makes the processing time available for particle filter estimation narrower which leds us to consider and estimate only horizontal positions while water currents are considered disturbances.

The sampling importance resampling (SIR) particle filter algorithm is composed by four steps [12], [13]: initialization, prediction (or propagation), measurement update and resampling. Here, we will present the particular implementation for single beacon localization. Let us consider the set of particles $\mathcal{X}_{k}=\left\{X_{k}^{1}, X_{k}^{2}, \ldots, X_{k}^{n}\right\}$, where $X_{k}^{i}=\left[x_{k}^{i}, y_{k}^{i}\right]^{T} \in \mathbb{R}^{2}$ and $n$ is the number of particles. Associated to these particles, a set of weights $\mathcal{W}_{k}=\left\{w_{k}^{1}, w_{k}^{2}, \ldots, w_{k}^{n}\right\}, w_{k}^{i} \in \mathbb{R}$, assign an importance to the corresponding particle. For initialization, the vehicle is stopped and the range is measured several times. The mean of sequential consistent measurement $\bar{R}_{i n i}$ is computed and each particle is sampled according to

$$
\left[\begin{array}{c}
x_{0}^{i} \\
y_{0}^{i}
\end{array}\right]=\left[\begin{array}{c}
\left(\bar{R}_{i n i}+r_{s}^{i}\right) \cos \left(\psi_{s}^{i}\right) \\
\left(\bar{R}_{i n i}+r_{s}^{i}\right) \sin \left(\psi_{s}^{i}\right)
\end{array}\right],
$$

where $r_{s}^{i} \sim \mathcal{U}\left(-\delta_{r}, \delta_{r}\right)$ and $\psi_{s}^{i} \sim \mathcal{U}(-\pi, \pi)$ are sampled from uniform probability distributions.
At each time step, each particle is propagated by deadreckoning similarly to (8) and (9). They differ only in an added sample $s_{k}^{i}$ from a user-defined distribution $p_{f}$, for propagation:

$$
X_{k+1}^{i}=f\left(X_{k}^{i}, \hat{\nu}_{k}\right)+s_{k}^{i} \text {. }
$$

Whenever a measurement of range is available, the update is performed. The weights are computed according to

$$
w_{k}^{i}=w_{k-1}^{i} q\left(R_{k} \mid X_{k}^{i}\right),
$$

where $q$ is an importance function that approach the likelihood. In this case the function $q=q\left(\|\| X_{k}^{i}\left\|\mid-R_{k}\right\|\right)$ is set to be a monotically decreasing, positive definite function of its argument. Our implementation makes use of an exponential function. After computing the weights, normalization follows: $w_{k}^{i}=w_{k}^{i} / \sum_{i}^{n} w_{k}^{i}$. The estimate of the state is then obtained through

$$
\hat{X}_{k}=\sum_{i=1}^{n} w_{k}^{i} X_{k}^{i}
$$

Note that this is not the only way to obtain the estimate. This approach was considered in [13]. Other implementations may contemplate only the most weighted particle, for example.

Due to their nature, after some iterations, it is common that particle filters weight a set of few particles considerably more than the remaining ones. In the single beacon localization problem, this happens when a small set of particles is iteratively in positions that are consistent with the range. Their weights will increase while the remaining ones will decrease. In such scenario, the reliability on the estimation decreases, once the estimation relies on this small set. This phenomenon is known as degenerancy. However, it can be solved by resampling. The method consists in "populating" the regions around the most weighted particles. This can be achieved by sampling from an accumulative weight function (see [12], for example) and setting all weights $w_{k}^{i}=1 / n$. Nevertheless, resampling should not be performed at each iteration but only when the degenerancy is high. A commonly used measure for degenerancy is the summation of the square of the weights [12], [13]. Thus, resampling has to be done whenever the following inequality holds:

$$
\frac{1}{\sum_{i}^{n} w_{k}^{i^{2}}}<N_{t h}
$$

where $N_{t h} \in[1, n]$ is a preset threshold.

Although particle filters provide an estimate based on vectors distributed in the space, it may diverge. Divergence, in this case, may be due to lack of coverage. Indeed, when the particles are resampled, they likely concentrate in a region of the space that may be too small which may originate lack of representativity for the measurements. Of course, this can be overcome by increasing the number of particles and tunning the user-defined distribution function $p_{f}$ such that the particles are propagated within a broader range. But, once again it has practical limitations related with computational capabilities. 


\section{Initialization}

Although the particle filters include a step for initialization, this is not yet solved for the EKF approach. Hence, in order to guarantee fast convergence of the Kalman filter to the real position, it is necessary to provide a fairly good guess of the initial position while reducing the values of the covariances of the corresponding variables. Unfortunately, the speed of convergence is not the only issue: without adequate initialization, the EKF will probably diverge and, as it is well-known, it would hardly recover. Baccou and Jouvencel [1] implemented an iterative mean square estimator for the initial position. However, the estimator relies on an accurate model for deadreckoning or accurate knowledge on vehicle velocities. Our implementation and simulation of least mean square method revealed us that the initialization is very sensitive to vehicle model uncertainties. On the other hand, least mean square method is optimal for Gaussian noise and acoustic pings suffer of non-Gaussian noise. Casey and Thomas [3] defined an intuitive estimator, based on least mean square errors, relying also on very accurate dead-reckoning.

Our approach makes use of the particle filter properties: a particle filter estimates the initial position of the vehicle which in turn is set as the initial estimate of the EKF. The initialization includes the following sequential steps:

1) The particle filter is initialized as stated in the previous subsection while the vehicle is stopped

2) The vehicle starts moving with an arbitrary orientation describing a line during 20-30 seconds (for $1 \mathrm{~m} / \mathrm{s}$ )

3) The vehicle describes an arc of a circle (tipically half of a circle)

4) The initial estimate of the Kalman filter is set with the value of the particle filter estimate.

Clearly, this does not ensure accurate initialization. However, the simulations showed that, in most cases, the initialization is fairly good and depends on the initial orientation, model parameters and currents.

Divergence of the Kalman can be easily detected through observation of the covariance matrix terms or large value of the norm of the water current velocities. In such case, the Kalman filter has to be re-initialized using the procedure presented above.

\section{Control}

If the actual position of the vehicle and the currents were known over the operation, the control law would be simple and would make the vehicle move in a straight line to the origin. However, it is easy to check that this linear motion lacks observability along the axis orthogonal to the motion when the position is being estimated. In fact, even with a very accurate initialization, the uncertainty along the refered axis would be high, which could make the filters diverge from the actual position. A method is described in [14] which makes it possible to compute the optimal control at each time step such that a given criterion of observability is maximized. However, the method is computationally expensive, since it implies the computation of Lagrange multipliers. Here, as in [1], we consider a curvilinear motion to approach the origin (beacon) (see fig. 2).

For control law derivation, let us consider the cylindrical coordinates where $\rho=\sqrt{x^{2}+y^{2}}$ is the distance of the vehicle to the origin projected in the horizontal plane where the vehicle is. Let $\psi_{p}=\arctan (y / x)$ be the angle of the segment that joins the vehicle and the beacon. As it was stated in section II, the control law has to stabilize the origin of $(x, y)$, which is equivalent to stabilize the origin of $\rho$. To derive the control law, we will use nonlinear Lyapunov theory. Our approach is based on backstepping (see [15]). Although a simple control law can be almost empirically derived, taking $u$ and $r$ as inputs of the system, Lyapunov theory and backstepping technique provide a framework to analyze and guarantee stability. We consider now the system:

$$
\begin{aligned}
\dot{\rho}= & u \cos \left(\psi_{p}-\psi\right)+v \sin \left(\psi_{p}-\psi\right)+v_{x} \cos \left(\psi_{p}\right) \\
& +v_{y} \sin \left(\psi_{p}\right) \\
\dot{\psi}= & r
\end{aligned}
$$

We define the Lyapunov function candidate as $V(\rho)=$ $1 / 2 \rho^{2}$ which satisfy the positive definiteness property. Its time derivative results

$$
\begin{aligned}
\dot{V}= & \rho \dot{\rho} \\
= & \rho\left[u \cos \left(\psi_{p}-\psi\right)+v \sin \left(\psi_{p}-\psi\right)\right. \\
& \left.+v_{x} \cos \psi_{p}+v_{y} \sin \psi_{p}\right] .
\end{aligned}
$$

In order to guarantee stability of the system, $\dot{V}$ has to be negative semi-definite. Asymptotic stability is ensured only if $\dot{V}<0$. Once $\rho>0, \dot{\rho}$ has to be negative semi-definite to achieve stability. Assuming that $u>0$, as it is the natural motion of the vehicle, from (17) follows that $\cos \left(\psi_{p}-\psi\right)<$ $0 \Rightarrow \psi \in] \psi_{p}+\pi / 2, \psi_{p}+3 \pi / 2[$.

Considering that the lateral velocity is small compared to the longitudinal one and the difference $\psi_{p}-\psi$ is small $(<\pi / 6)$,

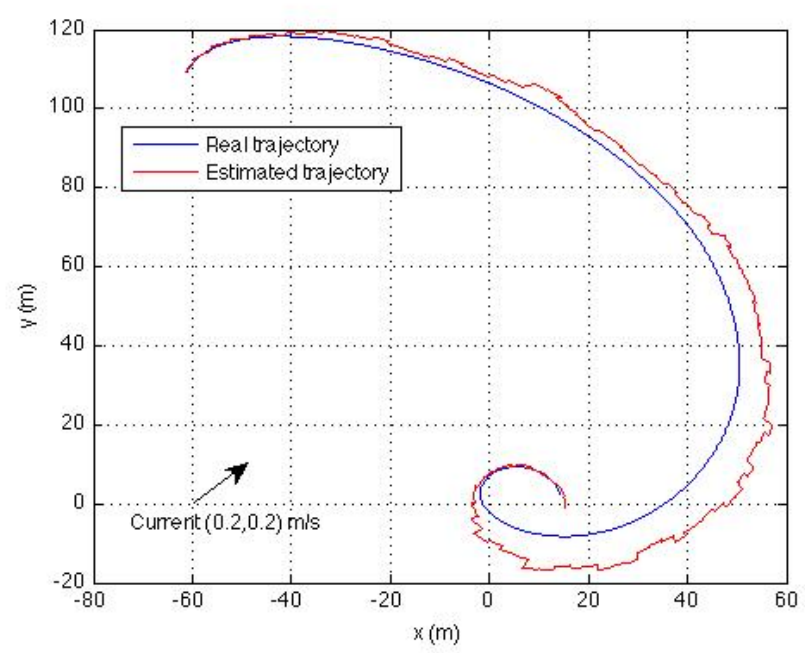

Fig. 2: Example of a homing trajectory. 
we can write

$$
\dot{\rho} \approx u \cos \left(\psi_{p}-\psi\right)+v_{x} \cos \psi_{p}+v_{y} \sin \psi_{p} .
$$

This assumption can be made since the yaw-rate $r$ is small. Otherwise, this approximation is no longer valid because $v$ grows with the increase of $u$ and $r$, in the particular case of MARES (see [6]). From this last equation, we derive easily the constraint on the forward velocity:

$$
u \cos \left(\psi_{p}-\psi\right)<-v_{x} \cos \left(\psi_{p}\right)-v_{y} \sin \left(\psi_{p}\right) .
$$

Suppose that $\psi=\xi\left(\psi_{p}\right)$ stabilizes the system (16) and makes the Lyapunov function satisfy the inequality $\dot{V} \leq$ $-W(\rho)$, where $W$ is a positive semi-definite function. The following expression satisfies the stabilization criteria:

$$
\xi\left(\psi_{p}\right)=\psi_{p}+\pi+\psi_{\text {off }},
$$

where $\left.\psi_{\text {off }} \in\right]-\pi / 2, \pi / 2[$ is an offset angle that makes possible the curvilinear approach. This offset makes the vehicle point to a virtual target on the side of the beacon. Defining the error variable $z=\xi-\psi$, the system (16) can be written as

$$
\begin{aligned}
\dot{\rho}= & u \cos \left(\psi_{p}-\xi+z\right)+v \sin \left(\psi_{p}-\psi\right) \\
& +v_{x} \cos \left(\psi_{p}\right)+v_{y} \sin \left(\psi_{p}\right) \\
\dot{z}= & r-\dot{\xi}
\end{aligned}
$$

In what follows, we will use the fact that, for each $z$, there exist a $\delta$ such that $\cos \left(\psi_{p}-\xi+z\right) \leq \cos \left(\psi_{p}-\xi\right)+\delta\|z\|$. Using $V^{\prime}=V+1 / 2 z^{2}$ as the new Lyapunov function, which is obviously positive definite, its time derivative is given by:

$$
\begin{aligned}
\dot{V}^{\prime} \approx & \rho\left[u \cos \left(\psi_{p}-\xi+z\right)\right. \\
& \left.+v_{x} \cos \left(\psi_{p}\right)+v_{y} \sin \left(\psi_{p}\right)\right]+z \dot{z} \\
\leq & -W(\rho)+\delta\|z\|+z(r-\dot{\xi}) .
\end{aligned}
$$

Choosing $r=\dot{\xi}-K z-K^{\prime} \operatorname{sign}(z)$, being $K^{\prime}$ chosen such that the term $\delta\|z\|$ is canceled, stability is ensured. Nevertheless, the stability will not be asymptotic due to the term $v \sin ($. neglected above but it can be achieved by imposing $u \rightarrow 0$ and $r \rightarrow 0$ as $\rho \rightarrow 0$.

So far in this subsection, we considered that the position of the vehicle is perfectly known. Indeed, it has to be estimated and uncertainties frequently grow when the vehicle describes some trajectories. Although in particle filter it is not trivial to infer about the confidence on the estimate, the Kalman filter provides an estimate of the uncertainty through its covariance matrix $P$. In general, the bigger the values of its entries, the less the confidence on the estimate is. In such scenarios it could be useful to change the trajectory. Here, the change of approach side could be enough in order to reduce the uncertainty on the estimate. Thus, our method makes use of the offset angle introduced in (20): whenever the elements of the covariance matrix grow above a preset threshold, the approach side is changed by simply setting $\psi_{o f f}=-\psi_{o f f}$ in the control law. But the switching frequency has to be bounded superiorly.

\section{RESUlts}

Implementation of our approach is demonstrated by realistic simulations using a six degree of freedom model of the dynamics, whose accuracy was already proved through comparisons in [6], and a non-gaussian time-of-flight noise. For simulation, we consider an exponential distribution whose domain is defined within the interval $[0, \infty[$. By sampling from this distribution, we try to approximate the previously observed behavior of the acoustic system.

In order to approach the real behavior of the dynamics, constraints and issues of the real operation, the on-board software is directly developed for the computational platform of the MARES AUV and tested through a simulator in Simulink (Matlab). Beyond validation of algorithms, this method enables performance evaluation and parameters tunning, as well. While the implementation code runs in its final version, the
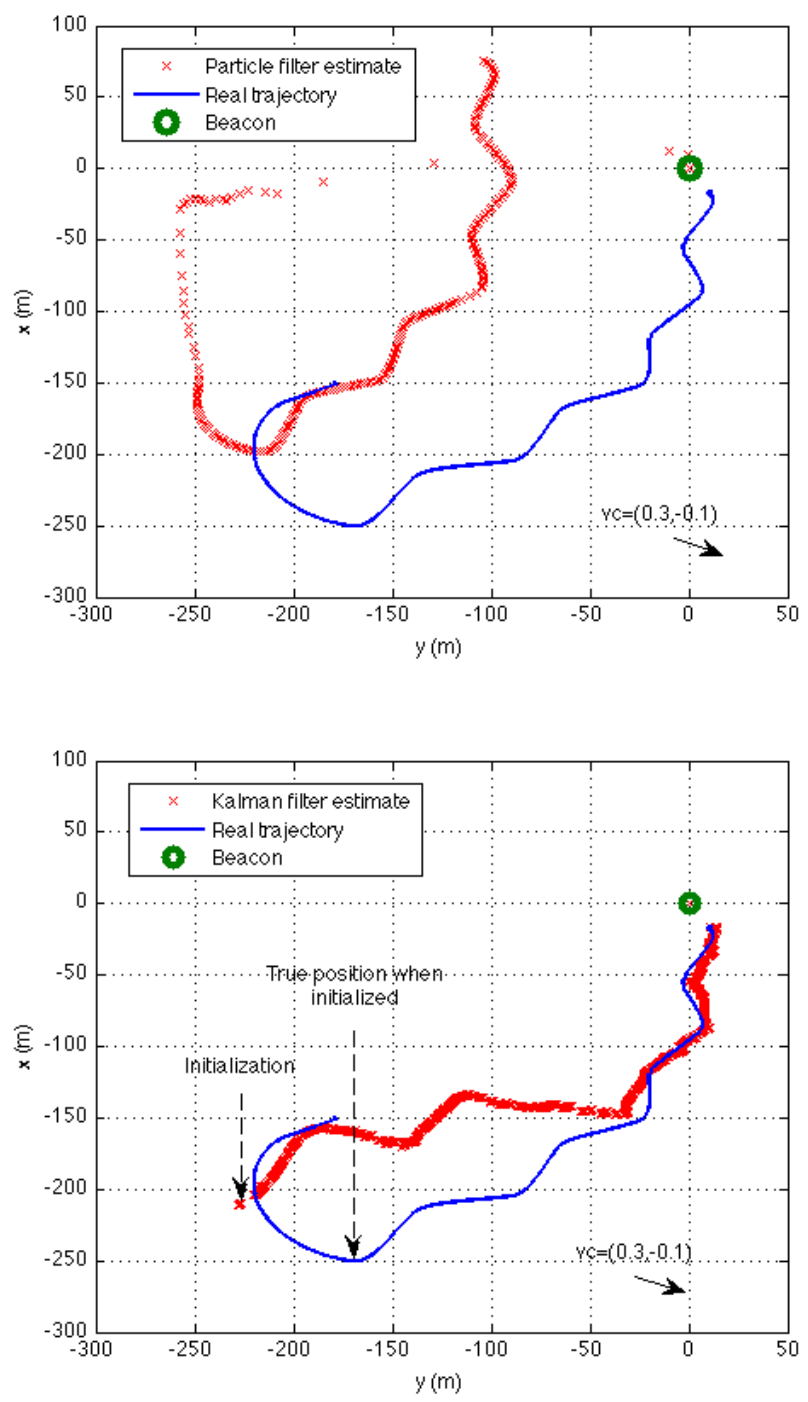

Fig. 3: Comparison between the particle filter and the extended Kalman filter estimates 
dynamics and the sensors are simulated accurately by the model developed in Simulink. A communication interface in the middle connects the two modules. Therefore the implementation code may be run in the local computer or in the AUV itself, since it can access to the local area network.

In this section, we show the results of both particle filter and Kalman filter approaches. We start by comparing the results in the same conditions and then follow with the analysis of the behavior of the best approach under different conditions.

\section{A. Comparison}

To find the best approach, the two estimators were implemented. Several simulations under different initial conditions were carried out and the final results were compared. However, here we will focus on a representative simulation. The results obtained from simulations with different initial positions and different water currents are similar to the one addressed here in terms of behavior. In order to make clear the algortihm followed for this simulation, let us recall the main steps:

1) the vehicle is set in a unknown random position, with an arbitrary heading

2) initialization is performed by the particle filter while describing a line during few seconds (30 seconds in this case) followed by an arc of circumference

3) Kalman filter is initialized with the last estimate of the particle filter after the initial trajectory

- whenever the diagonal elements of the covariance matrix $P$ grow above a preset threshold (150 for the simulation), the approach side is switched

4) the homing process ends when the vehicle is close to the beacon ( 20 meters in this simulation)

The homing trajectory shown in fig. 3 starts at point $(x, y)=(-150,-180)$ with an arbitrary heading. The water current variables were set to $\left(v_{x}, v_{y}\right)=(0.3,-0.1)$. The controller takes the estimations of the Kalman filter to generate the trajectory, moving at a constant forward speed of $1 \mathrm{~m} / \mathrm{s}$. In the fig. 3a, we show the result of the estimation of the particle filter. The estimate starts at $(0,0)$ and then tends to approach the real position. However, it can be seen that the particle filter diverges after some steps. The cause of this divergence is the lack of coverage of the particles: after re-sampling several times, they occupy a small area and their propagation is not enough to prevent divergence. After divergence, the particles are basically driven by dead-reckoning. Propagation can be made larger with an adequate choice of the distribution $p_{f}$ from which the "noise" of propagation is sampled. But this approach has critical consequences, specially for filters with small number of particles, since they may be propagated too far from the real position. This issue could be overcome choosing a larger number of particles and including the estimate of the currents in the state vector to be estimated. Our experience tells us that, for a number of particles between $n=100$ and $n=1000$, the behavior remains similar. Therefore, a larger number is needed and it has to be even much larger if the water current variables are added. However, we were not able to do so because of the computational constraints.
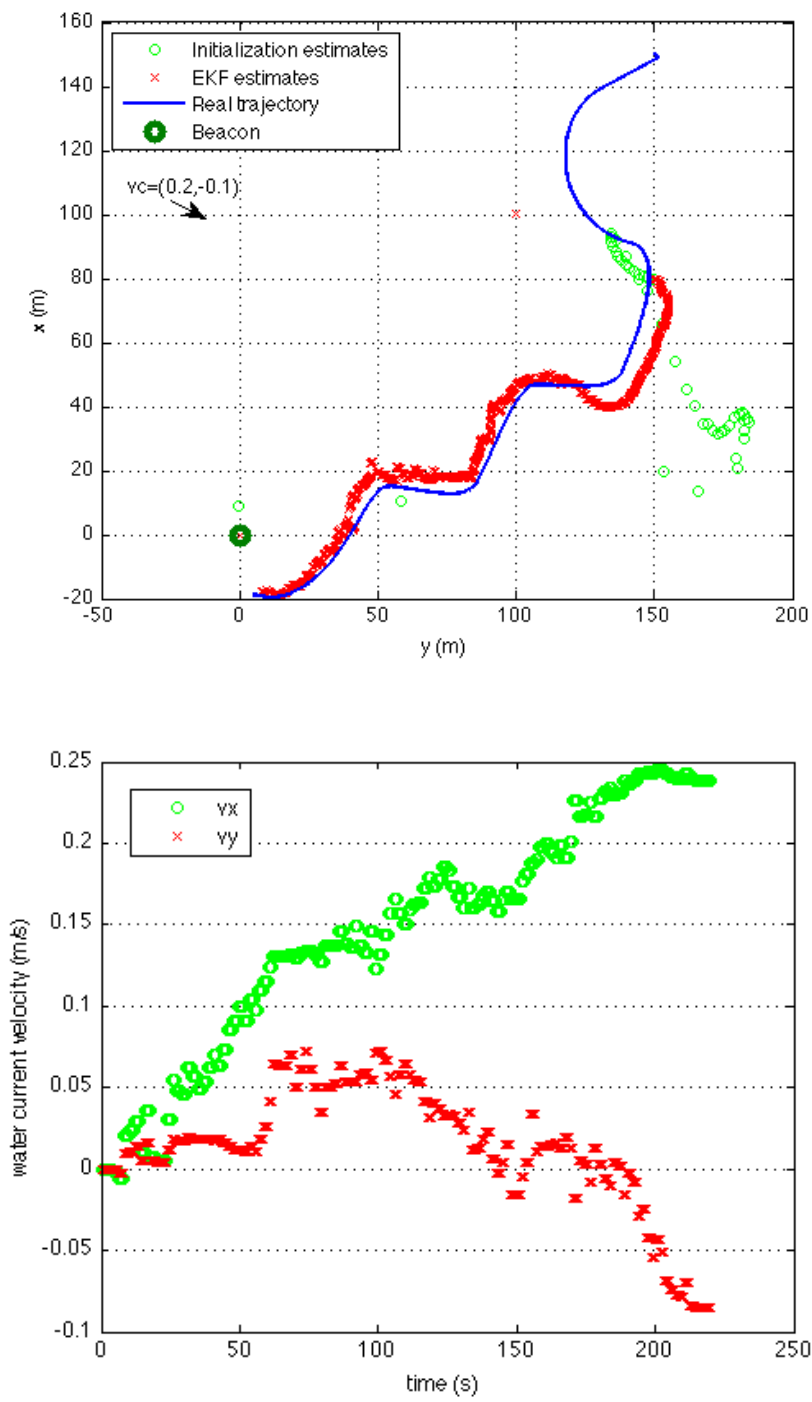

Fig. 4: Position and current velocity estimations

On the other hand, the fig. $3 \mathrm{~b}$ shows that the Kalman filter converges to the true position of the vehicle, even with a poor initialization. Some terms of the covariance matrix are above the preset threshold which make the controller switch the approach side several times. Most of the simulation showed that the extended Kalman filter approach provides the best results and the overall estimation is robust when it is initialized with the particle filter. Next, we will focus on the results of this approach.

\section{B. EKF approach}

The results shown above led us to conclude that the extended Kalman filter approach is the better than the particle filter one. Moreover, the use of the covariance matrix to infer about the confidence on the estimates provides to the system robustness and ability to take decisions according to. For example, re-initialization is specially appreciated in this context. 

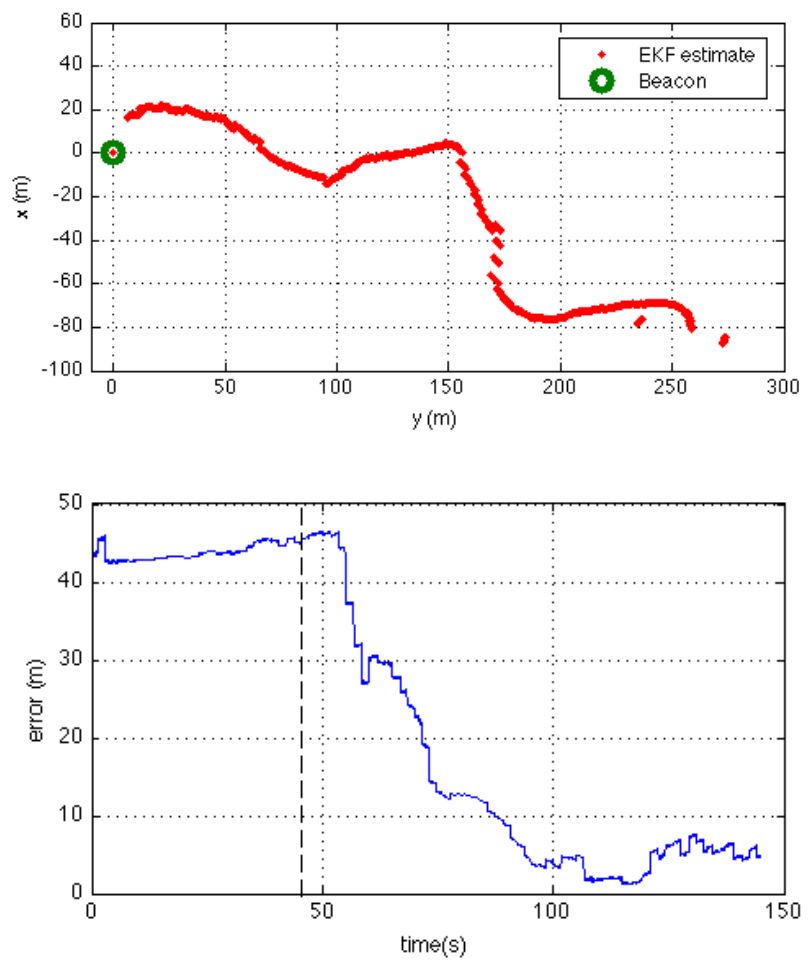

Fig. 5: Homing at $2 \mathrm{~m} / \mathrm{s}$

In the following simulation, the vehicle is set at $(x, y)=$ $(150,150)$ with current velocity $\left(v_{x}, v_{y}\right)=(0.2,-0.1)$. In fig. 4 we show the estimation of the position and of the current component velocities. As we can see, the estimation of the these latter is slow and erroneous almost of the time. This shows that the estimation of these variable is not critical.

In the present approach, the errors in the dead-reckoning model of (8) and (9) are reflected on the estimation of the current velocity. This fact is easy to explain: imagine that the vehicle is moving in a straight line and the estimated forward velocity is inaccurate. The difference between the dead-reckoned and real forward velocities will be directly reflected on the estimation.

Finally, we show the result of another homing mission with different surge velocity $(u=2 \mathrm{~m} / \mathrm{s})$ in the presence of voluntary model uncertainty and current $\left(v_{x}, v_{y}\right)=(0.4,0)$ in fig. 5. The surge drag coefficient was set to be $30 \%$ less than its actual value. This fact together with non modeled current affect the initialization. In this case, the fig. 5b shows that the initialization is made with an error of $43 \mathrm{~m}$. However, one can remark that the estimation the EKF recover from this error. The dashed line indicates the moment at which the approach side is switched for the first time $(\sim 45 \mathrm{~s})$ and we can see that observability is improved in such a way that the error decrease quickly. These correction appear in fig. 5a like "discontinuities" (roughly speaking) after the first curve.

These results reveal that this approach is robust even in the presence of model uncertainties and possible inaccurate initializations.

\section{CONCLUSION AND FUTURE WORK}

In this paper we presented a complete single beacon navigation solution. Although we focused on the homing problem, the application of the concepts showed here are easily transposed to other problems. We first described the main questions to be approached, presenting the MARES autonomous underwater vehicle as well as the localization system. We tackled the problem of localization by developing two estimators of different nature. Particle filter is a promising estimator that does not make assumptions on the noise, however the number of particles has to be large to ensure robustness and good performances. On the other hand, the extended Kalman filter make some assumption on the kind of noise and need for initialization but it is generally less exigent in terms of computational requirements. A method using both filters was proposed: the particle filter estimates the initial position which is used by the extended Kalman filter as the first estimate. Control questions could not be decoupled from estimation in this paper, since observability is directly related with the trajectory. We derived control law such that the trajectory changes according to the confidence on the estimation. Finally, we showed the results of the implementation by means of accurate simulations. While the performances of the extended Kalman filter approach demonstrated robustness and accuracy, the approach that use only the particle filter results frequently in divergences of the estimates.

At time of writing this paper, experiments are being conducted. We expect to test this solution under different conditions such as position, distance water current. In a future implementation, a gradient-based approach for homing is envisioned, extending the concept of beacon to other domains like environment monitoring.

\section{REFERENCES}

[1] P. Baccou and B. Jouvencel, "Homing and navigation using one transponder for auv, postprocessing comparisons results with long baseline navigation," in Robotics and Automation, 2002. Proceedings. ICRA 'O2. IEEE International Conference on, vol. 4, 2002, pp. 4004 - 4009 vol.4.

[2] P. Newman and J. J. Leonard, "Pure range-only subsea slam." in Proceedings of the IEEE International Conference on Robotics and Automation, Taiwan, September 2003.

[3] T. Casey, B. Guimond, and J. Hu, "Underwater vehicle positioning based on time of arrival measurements from a single beacon," in OCEANS 2007, sept. 2007, pp. $1-8$.

[4] E. Olson, J. Leonard, and S. Teller, "Robust range-only beacon localization," in Autonomous Underwater Vehicles, 2004 IEEE/OES, 17-18 2004, pp. $66-75$.

[5] G. Rui and M. Chitre, "Cooperative positioning using range-only measurements between two auvs," in OCEANS 2010 Sydney, Australia, May 2010.

[6] B. Ferreira, A. Matos, N. Cruz, and M. Pinto, "Modeling and Control of the MARES Autonomous Underwater Vehicle," MARINE TECHNOLOGY SOCIETY JOURNAL, vol. 44, no. 2, Sp. Iss. SI, pp. 19-36, MARAPR 2010.

[7] N. Santos, A. Matos, and N. Cruz, "Navigation of an Autonomous Underwater Vehicle in a Mobile Network," in OCEANS 2008, VOLS 1-4, ser. OCEANS-IEEE, IEEE. 345 E 47TH ST, NEW YORK, NY 10017 USA: IEEE, 2008, Proceedings Paper, pp. 1196-1200, OCEANS 2008 Conference, Quebec, CANADA, SEP 15-18, 2008. 
[8] N. A. Cruz and A. C. Matos, "The MARES AUV, a Modular Autonomous Robot for Environment Sampling," in OCEANS 2008, VOLS $1-4$, ser. OCEANS-IEEE, IEEE. 345 E 47TH ST, NEW YORK, NY 10017 USA: IEEE, 2008, Proceedings Paper, pp. 1996-2001, OCEANS 2008 Conference, Quebec, CANADA, SEP 15-18, 2008.

[9] T. I. Fossen, Guidance and control of ocean vehicles. John Wiley \& Sons, Chichester, 1995.

[10] T. Prestero, "Verification of a six-degree of freedom simulation model for the remus autonomous underwater vehicle," Master's thesis, Massachussets Institute of Technology, 2001.

[11] A. Gelb, Applied optimal estimation. MIT Press, 1974.

[12] M. Arulampalam, S. Maskell, N. Gordon, and T. Clapp, "A tutorial on particle filters for online nonlinear/non-Gaussian Bayesian tracking," IEEE TRANSACTIONS ON SIGNAL PROCESSING, vol. 50, no. 2, pp. 174-188, FEB 2002.

[13] F. Gustafsson, F. Gunnarsson, N. Bergman, U. Forssell, J. Jansson, R. Karlsson, and P. Nordlund, "Particle filters for positioning, navigation, and tracking," IEEE TRANSACTIONS ON SIGNAL PROCESSING, vol. 50, no. 2, pp. 425-437, FEB 2002.

[14] H. Feder, J. Leonard, and C. Smith, "Adaptive mobile robot navigation and mapping," INTERNATIONAL JOURNAL OF ROBOTICS RESEARCH, vol. 18, no. 7, pp. 650-668, JUL 1999.

[15] H. K. Khalil, Nonlinear Systems. Prentice Hall, 2002. 